\title{
Factores relacionales familiares y el desarrollo de distimia en los adolescentes
}

\section{Family relational factors and the development of dysthymia in adolescents}

1 Verónica Adriana Freire Palacios (iD) https://orcid.org/0000-0001-9493-6552. Universidad Técnica de Ambato, Facultad de Ciencias Humanas y de la Educación, Carrera de Educación Básica, Ambato, Ecuador va.freire@uta.edu.ec

2 Carlos Alfredo Hernández Dávila (iD) https://orcid.org/0000-0002-2526-5051 Universidad Técnica de Ambato, Facultad de Ciencias Humanas y de la Educación, Carrera de Educación Básica, Ambato, Ecuador ca.hernandez@uta.edu.ec

3 Carmen Dolores Chávez Fuentes (iD) https://orcid.org/0000-0002-5237-9636. Universidad Técnica de Ambato, Facultad de Ciencias Humanas y de la Educación, Carrera de Educación Básica, Ambato, Ecuador cd.chavez@uta.edu.ec

4 Héctor Daniel Morocho Lara (iD) https://orcid.org/0000-0003-3107-6238 Universidad Técnica de Ambato, Facultad de Ciencias Humanas y de la Educación, Carrera de Educación Básica, Ambato, Ecuador hd.morocho@uta.edu.ec

Cítese: Freire Palacios, V. A., Hernández Dávila, C. A., Chávez Fuentes, C. D., \& Morocho Lara, H. D. (2023). Factores relacionales familiares y el desarrollo de distimia en los adolescentes. $\quad$ ConcienciaDigital, 6(1.4), 950-969. https://doi.org/10.33262/concienciadigital.v6i1.4.2043

CONCIENCIA DIGITAL, es una Revista Multidisciplinar, Trimestral, que se publicará en soporte electrónico tiene como misión contribuir a la formación de profesionales competentes con visión humanística y crítica que sean capaces de exponer sus resultados investigativos y científicos en la misma medida que se promueva mediante su intervención cambios positivos en la sociedad. https://concienciadigital.org _

La revista es editada por la Editorial Ciencia Digital (Editorial de prestigio registrada en la Cámara Ecuatoriana de Libro con No de Afiliación 663) www.celibro.org.ec 


\section{Palabras}

claves:

Psicología,

Factores

Relacionales,

Familia,

Distimia,

Adolescentes
Keywords:

Psychology,

Relational

Factors,

Family,

Dysthymia,

Adolescents
Resumen

Introducción: La distimia es una condición poco estudiada y al ser considerada como un trastorno depresivo mayor es un problema de salud pública, debido a las consecuencias y manifestaciones que afectan no solo al individuo sino a todo el grupo familiar y social, puesto que involucra alteraciones emocionales graves y duraderas. Objetivo: Determinar los factores relacionales familiares asociados al desarrollo de la distimia en adolescentes. Metodología: Estudio correlacional causal, de tipo no experimental, con un muestreo no probabilístico intencional; se contó con una muestra de 98 participantes, los instrumentos empleados fueron la Escala Birleson Modificada conformada por 21 preguntas, con una puntuación tipo Likert de 0 a 2 para identificar la presencia o ausencia de distimia, FACES III posee 20 ítems, con una escala de puntuación tipo Likert de 5 alternativas para evaluar la cohesión y la adaptabilidad y Escala de Clima Social estructurada por 90 ítems, que valora las características socio ambientales de la familia, se utilizó la prueba no paramétrica del chi cuadrado (x2), permitiendo estimar la relación entre los factores relacionales familiares y la distimia. Resultados: En relación con la distimia se presentaron en el 59.2\% de los adolescentes. El clima sociofamiliar se asocia a la presencia de distimia $(p=0.017)$, el $21.8 \%$ de los adolescentes con distimia tuvieron un clima familiar "Malo", mientras que el $25.6 \%$ de los que no tuvieron distimia tuvieron un clima familiar "Bueno". El grado de adaptación de la familia no se relaciona a la distimia $(\mathrm{p}=0.283)$. Conclusión: los factores relacionales familiares como: la desatención, los conflictos de independencia, las dificultades emocionales con los padres, el conflicto conyugal, eventos vitales negativos en el entorno familiar, antecedente de intento suicida de algún familiar, violencia familiar se encuentran relacionados al desarrollo de la distimia en más de la mitad de los adolescentes.

\section{Abstract}

Introduction: Dysthymia is a poorly studied condition and being considered as a major depressive disorder is a public health problem, due to the consequences and manifestations that affect not only the individual but the entire family and social group, since it involves alterations serious and long-lasting emotional Objective: To determine the family relational factors 
associated with the development of dysthymia in adolescents. Methodology: Causal correlational study, of a nonexperimental type, with an intentional non-probabilistic sampling; There was a sample of 98 participants, the instruments used were the Modified Birleson Scale made up of 21 questions, with a Likert-type score from 0 to 2 to identify the presence or absence of dysthymia, FACES III has 20 items, with a scale of Likert-type score of 5 alternatives to evaluate cohesion and adaptability and Social Climate Scale structured by 90 items, which assesses the socio-environmental characteristics of the family, the non-parametric chi-square test (x2) was used, allowing to estimate the relationship between family relational factors and dysthymia. Results: In relation to dysthymia, they occurred in $59.2 \%$ of adolescents. The socio-family climate is associated with the presence of dysthymia $(p=0.017), 21.8 \%$ of adolescents with dysthymia had a "Bad" family climate, while $25.6 \%$ of those who did not have dysthymia had a family climate " Okay". The degree of adaptation of the family is not related to dysthymia ( $p=0.283$ ). Conclusion: family relational factors such as inattention, independence conflicts, emotional difficulties with parents, marital conflict, negative life events in the family environment, a history of suicide attempt by a relative, family violence are related to the development of dysthymia in more than half of adolescents.

\section{Introducción}

En la actualidad la depresión se considera un problema de salud pública, tanto a nivel mundial como en nuestro país, debido a las consecuencias y manifestaciones que afectan no solo al individuo sino a todo el grupo familiar y social, puesto que involucra alteraciones emocionales graves y duraderas. Se estima que 1 de cada 33 niños y 1 de cada 8 adolescentes sufre de depresión en el mundo, hallando una prevalencia que puede variar del $0.7 \%$ al 11\%. En países como Japón la frecuencia es del 3\% alcanzando, un $16.9 \%$ en Estados Unidos (Wan, 2012). En países como Colombia (Medellín), se identificó que el $12.1 \%$ de los adolescentes tuvo el diagnóstico de depresión, de los cuales, el $9.3 \%$ fue reconocido en hombres y el $13.9 \%$ en mujeres, señalando diferencias significativas entre ambos sexos (Hoyos et al., 2012). En Chile las tasas de depresión son de un $2 \%$ en la niñez y de 4 a $8 \%$ en la adolescencia, aumentando a medida que avanza el periodo evolutivo y dependiendo principalmente del sexo del adolescente (más prevalente en mujeres que en 
hombres) (Ministerio de Salud Gobierno de Chile, 2013).

En el Perú, según lo identificado por la vigilancia de salud mental desarrollado a nivel de la Dirección de la Red de Salud Lima Cuidad (DRSLC), el 24.4\% de los adolescentes tiene problemas depresivos de los cuales el 5.6\% son adolescentes de sexo femenino y el 18.8\% masculino. Sin embargo el Ministerio de Salud Pública, Gobierno del Perú ( 2014), manifiesta una situación sui generis en comparación con otras realidades y el primer problema de salud mental en nuestro medio, no sólo por el aumento de casos sino también por las consecuencias, en este grupo poblacional, tales como: malestar clínico, ausentismo escolar y bajo rendimiento, riesgo de abuso de sustancias, conductas antisociales, conductas riesgosas sexuales y en la alimentación, intentos suicidas y suicidios.

La distimia es una de las formas de manifestación de la depresión, tan extendida que por ejemplo en los Estados Unidos el 1.35 de cada 25 habitantes la padece y en América Latina y en el Caribe la sufre el 1,7\% de la población, según la Organización Panamericana de la Salud (OPS, 2012). En la actualidad está cobrando nuevas víctimas de manera peligrosamente silenciosa, pues casi sin darnos cuenta, está presentándose en una población vulnerable en la que muchas de las veces se le puede confundir con las conductas propias de su edad: "los adolescentes".

El DSM-5 define a la distimia (trastorno depresivo persistente) como un estado de ánimo en el que, específicamente, el niño y/o el adolescente se encuentra irritable y deprimido durante la mayor parte del día, teniendo una duración como mínimo de un año (American Psychiatric Association (APA, 2014).

Por otro lado, Pérez (2020) en su trabajo señala que la distimia es un trastorno mental caracterizado por presentar comportamientos depresivos con un nivel de severidad más bajo, pero de manera más persistente y con una alta probabilidad de convertirse en crónicos si no son tratados a tiempo. De la misma forma que la depresión, la distimia produce consecuencias graves que se deben atender y dar una intervención oportuna para evitar complicaciones crónicas para la salud mental tanto del individuo como de la familia.

Es importante manifestar que este trastorno no viene solo sino que, en el caso de niños y adolescentes con distimia, tanto al inicio como durante su curso natural, se asocia a otros trastornos psiquiátricos y conductas patológicas tales como: trastornos de ansiedad (40\%), problemas de conducta o drogadicción (30\%), déficit de atención con hiperactividad (ADHD, 30\%), enuresis o encopresis (15\%), ocasionando el incremento de la mortalidad, la tasa de suicidios, homicidios, comportamientos agresivos y consumo de drogas. El siguiente dato no solo es revelador sino también significativo: "El trastorno distímico representa un riesgo de intentos suicidas, con tasas que varían entre $9.5 \%$ a $19 \%$ en la población adolescente" (Nobile et al., 2003). 
Estas estadísticas revelan que la adolescencia es una etapa crítica para la aparición de la distimia. Ante esto es menester considerar que diversos autores señalan que el entorno familiar es el principal factor que puede influenciar en el desarrollo de dicho trastorno, puesto que el adolescente pasa el mayor tiempo con su familia tanto en horas ( 4.8 horas al día) como en años (aproximadamente 19 años de su vida), datos que direccionarían a evaluar los factores relacionales de la familia que pudieran influenciar en la aparición de dicho trastorno en la vida de los adolescentes y jóvenes (Galicia et al., 2009).

Diversas teorías abordan el tema de la depresión en general y de la distimia en particular desde sus principios y perspectivas individuales, por ejemplo, la teoría psicoanalítica refiere que la depresión es producto de la pérdida del interés por el mundo externo que se traduce en una conducta inhibida en la persona, inhibición que suele ser generalizada a todas sus facetas. Básicamente se trata de un sentimiento que se une al descenso de la autoestima. Según la teoría conductista se enfoca principalmente en la teoría de la indefensión o desesperanza aprendida, es decir, que cuando una persona ha tenido experiencias en el pasado que le han sido desagradables y frente a las cuales no ha podido luchar, puede desarrollar un estilo de pensamiento en el que se convence a sí mismo de que toda situación desagradable que pueda experimentar estará fuera de su control. Por otro lado, la teoría cognitivo-conductual, interpreta a la depresión como una consecuencia de las distorsiones que una persona puede desarrollar al procesar cognitivamente la información que obtiene de lo que le rodea, es decir, es un sentimiento elaborado de manera mental y que tiene un correlato en la realidad a través del comportamiento.

Desde una perspectiva sistémica, los síntomas depresivos que presenta un adolescente no serían atribuibles solo a él, sino también a las diversas interrelaciones que establece con su medio, principalmente con su entorno doméstico; por ello resulta importante observar y valorar cómo se expresa la dinámica relacional entre el joven y su mundo familiar, buscando identificar qué factores relacionales puedan estar siendo responsables de la aparición del trastorno depresivo. Podríamos observar, por ejemplo: los problemas de comunicación entre padres e hijos, la conflictividad, la hostilidad, el excesivo control conductual de los padres, la baja experimentación de afecto y de cercanía, etc.

Con la experiencia clínica se ha observado que muchos de los adolescentes por diferentes motivos, presentan rasgos de distimia como problema principal o como una comorbilidad de otros problemas psicológicos. Además al extender la pesquisa con vistas al contexto familiar del que proceden dichos jóvenes, se evidencia que ellos vienen de hogares difíciles en el que se respira una atmósfera relacional caracterizada por un clima emocional frio e hipercrítico, sazonado con ciertos grados de violencia, abierta o encubierta; sospechando que pueden existir pautas relacionales distorsionadas en la familia que estarían asociados a la emergencia de aquellos síntomas distímicos, que se hace necesario investigar. 
Lo presentado en este trabajo, al igual que lo encontrado en la literatura, indica que ciertos elementos del clima relacional familiar, como la dinámica familiar alterada, el autoritarismo, la falta de cohesión familiar, la falta de apoyo intrafamiliar, la irritabilidad y la agresividad de los padres, entre otros, influirían en la sintomatología depresiva en los adolescentes y jóvenes. Más allá de intentar abrir nuevas líneas de estudio, el reconocimiento de las implicancias que tienen las interacciones familiares en el desarrollo psicosocial de los adolescentes y jóvenes, y el impacto de ello en su estado emocional, es de gran relevancia y no sólo debe quedarse a nivel de investigación, ya que ésta es sólo el primer paso para el reconocimiento de las problemáticas sociales.

\section{Metodología}

Diseño: El nivel de estudio fue correlacional causal, puesto que se ha buscado establecer el grado de asociación entre las variables, es decir se investigó si existe relación significativa entre los factores relacionales familiares y la distimia. Ha sido de tipo no experimental, porque no existió manipulación de alguna de las variables, solo se observó cómo se desenvuelven en la realidad, es decir se recolectaron los datos sin que hubiera intervención de los investigadores sobre alguna de ellas.

Muestra: La población de estudio que se consideró estuvo constituida por 120 adolescentes del quinto año de secundaria de la I.E. 3055 "Túpac Amaru" de 15 a 17 años, bajo los siguientes criterios de inclusión: que posterior a la aplicación de la Escala Birleson modificada, presentaron un diagnóstico confirmatorio de distimia según los criterios del DSM-V; para el caso de este estudio la muestra es no probabilístico intencional se contó con una muestra de 98 adolescentes.

\section{Escala de Clima Social (FES)}

Esta escala considera las rasgos o peculiaridades socio ambientales de las familias, evaluando las relaciones interpersonales entre los miembros de la familia, los aspectos de desarrollo que tienen mayor importancia y su estructura básica (Osorio, 2019). La confiabilidad del instrumento tiene un Alpha de Cronbach de 0.78 y la validez reporta puntajes $r<0.80$.

Esta escala consta de 90 ítems, conformada por tres dimensiones y a la vez éstas poseen subescalas. La primera dimensión es Relaciones posee tres subescalas que evalúan el grado de cohesión, expresividad y conflicto; la segunda dimensión es desarrollo que está compuesta por seis subescalas que evalúan la importancia para la familia de ciertos procesos de desarrollo personal, éstas son: autonomía, actuación, intelectual-cultural, social-recreativo, moralidad y religiosidad y por último la tercera dimensión es estabilidad que se encarga de evaluar la estructura, organización de la familia y el grado de control que ejercen unos miembros sobre otros, ésta se conforma por dos subescalas: organización y control. 
La puntuación máxima de la escala es de 90 puntos y la mínima de 0 puntos; agrupados en 10 factores (cada uno de 9 ítems que tienen una puntuación máxima de 9 y mínima de $0)$.

Al tratarse de una versión española adaptada a nuestra realidad, se hizo necesario realizar algunos cambios para establecer la correspondencia lingüística de los términos en varios de los ítems, ya que eran expresiones y vocablos típicamente españoles. No se realizaron modificaciones en los contenidos asegurando de esta manera, la equivalencia conceptual (Cassullo, 1998).

\section{Escalas de evaluación de la adaptabilidad y cohesión familiar III (FACES III)}

Fundado en el patrón circumplejo de sistemas conyugales y familiares de Olson. Ha sido desarrollado con el objetivo de viabilizar la conexión entre la práctica clínica, la teoría y la investigación con familias. Reúne las tres dimensiones importantes del funcionamiento familiar (la capacidad del sistema familiar para enfrentar y superar cada una de las etapas del ciclo vital y las crisis por las que atraviesa): adaptabilidad, cohesión y comunicación. Este modelo propició la elaboración de una serie de instrumentos de evaluación entre los cuales se incluye FACES III, que es un instrumento de auto aplicativo que valora dos principales funciones a través de 20 ítems. Tiene un alto grado de confiabilidad y es de fácil aplicación e interpretación, se ha establecido como una herramienta con óptimas proyecciones de aplicación en la experiencia clínica tanto como en la investigación académica. Tiene con una escala de puntuación tipo Likert que encierra cinco alternativas: nunca o casi nunca, pocas veces, a veces, con frecuencia y casi siempre, de tal manera que la puntuación posible para cohesión y la adaptabilidad es de 10 a 50.

La confiabilidad del instrumento por alfa de Cronbach es de 0.87. El cuestionario se administra a individuos de más de 10 años, que sean miembros de una familia con hijos y alfabetos. Se pide al interrogado que conteste las interrogaciones considerando cada proposición en relación con su realidad familiar.

Es necesario aclarar que este instrumento no evalúa de manera directa la funcionalidad familiar, pues únicamente, como ya se ha referido, evalúa el grado de las primeras dimensiones referidas: cohesión y adaptabilidad; la comunicación se valora a través de otro instrumento (ENRICH).

$\checkmark 10$ preguntas evalúan la cohesión familiar.

$\checkmark 10$ preguntas evalúan la adaptabilidad familiar.

La FACES III esta subdividida en dos dimensiones:

Niveles de cohesión: está categorizado de la siguiente manera: 
- No relacionada o dispersa: 10 a 34 pts.

- Semi relacionada o separada: 35 a 40 pts.

- Relacionada o conectada: 41 a 45 pts.

- Aglutinada: 46 a 50 pts.

> Niveles de adaptabilidad: está categorizado de la siguiente manera:

- Rígida: 10 a 19 pts.

- Estructurada: 20 a 24 pts.

- Flexible: 25 a 28 pts.

- Caótica: 29 a 50 pts.

Los puntajes obtenidos mediante FACES III han propiciado la clasificación de las familias al considerar tres categorías: balanceadas, en rango medio y extremas. Se ha establecido la hipótesis de que las familias extremas presentan mayor riesgo de disfunción, lo cual ha sido corroborado en la experiencia clínica de diversos investigadores. Aunque recientes publicaciones han dado a conocer FACES IV y sus seis escalas (dos balanceadas y cuatro no balanceadas) es necesario considerar que el proceso de traducción, retro traducción, transculturación, aplicación a muestras heterogéneas de familias, evaluación de consistencia interna de cada escala, así como la evaluación de su validez de constructo no se han efectuado todavía en español.

\section{Escala de Birleson modificada}

Esta es una escala que fue elaborada teniendo como parámetro referencial la Escala de Depresión de Zung que evalúa la depresión en general y de manera específica el trastorno distímico (síntomas afectivos, cognitivos y somáticos). La consistencia interna es de moderada a buena y menor para pacientes internados en centros hospitalarios.

La fiabilidad test-retest es buena. En general, hay divergencias de género, las adolescentes puntúan más alto que los adolescentes. Aunque la validez, en general, es apropiada, presenta una pobre validez discriminante. Las ventajas más importantes de la escala de Birleson implican la posibilidad de un acomodo para niños y adolescentes, con un lenguaje muy simple, brevedad de las proposiciones, facilidad de uso y de puntuación (Rafael, 2007). La presente versión modificada está conformada por 21 preguntas, las cuales están diseñadas como actitudes con una escala de puntuación tipo Likert de 0 a 2. Para medir el resultado de distimia se utiliza la suma de los veintiún ítems. Una puntuación mayor significa una depresión más profunda.

\section{Procedimiento de recolección y análisis de información}

El proceso de recolección de los datos se realizó en dos fases: 
Primera fase: se identificaron a los adolescentes con diagnóstico o no, de distimia; este diagnóstico se realizó mediante la aplicación de la escala Birleson modificada, a todos los alumnos del nivel secundario, con la finalidad de identificar los casos de distimia, sin depresión mayor; cabe resaltar que según el DCM V, el diagnóstico confirmatorio de distimia se da luego de un año de haber realizado el primer diagnóstico; por lo cual, solo se considerarán adolescentes con distimia a aquellos que en la primera evaluación realizada el mes de diciembre del año 2015 (al finalizar las clases escolares) hayan sido diagnosticados con distimia y que en el último test de Birleson realizado hayan repetido este diagnóstico.

Segunda fase: para indagar sobre los factores asociados a este trastorno, desde un enfoque sistémico familiar, se aplicó un cuestionario previamente validado que permitió identificar después del análisis, el grado la asociación entre ambas variables de estudio (factores familiares relacionales y la distimia); este cuestionario ha sido proporcionado, tanto a alumnos con diagnóstico de distimia, como a los que no presentaron depresión, ya que según las características de los estudios correlacionales, necesitaron tener una muestra que presente el trastorno (distimia) y otra que no la presente, y en ambas muestras se analizaron qué factores relacionales se encuentran asociados y el grado de asociación con los factores relacionales familiares y cada una de las dimensiones.

Ambas fases se realizaron durante la evaluación psicológica que pasan todos los adolescentes de esta institución educativa, en cada año de estudio (primero, segundo, tercero, cuarto y quinto de secundaria). Cabe resaltar que esta encuesta ha sido de carácter anónimo.

\section{Análisis de los datos}

La información recogida de la ficha de recolección de datos ha sido expuesta a un control de calidad, seleccionando aquellos que estén correctamente llenados, luego ha sido foliado e ingresado a una base de datos elaborada en el programa IBM Statistics SPSS versión 22.0 para su análisis. Se realizaron dos tipos de análisis, uno descriptivo y otro inferencial.

Análisis descriptivo o univariado: para las variables cuantitativas se estimaron medidas de tendencia central y medidas de dispersión y para las variables cualitativas se estimaron frecuencias absolutas y relativas.

Análisis inferencial: se utilizó la prueba no paramétrica del chi cuadrado $\left(\mathrm{x}^{2}\right)$, puesto que las variables que analizamos han sido cualitativas; la cual permitió estimar la relación entre los factores relacionales familiares y la distimia en los adolescentes. 


\section{Resultados}

Los resultados del análisis descriptivo han sido presentados en tablas simples y los resultados del análisis inferencial en tablas de contingencia.

\section{Tabla 1}

Datos generales de los adolescentes del nivel secundario

\begin{tabular}{llc}
\hline \multicolumn{1}{c}{ Edad } & \multicolumn{2}{c}{ Media \pm DE } \\
& \multicolumn{2}{c}{$15.76 \pm 0,99(14-19)$} \\
& $\mathrm{N}$ & $\%$ \\
Sexo & 57 & $58.2 \%$ \\
Femenino & 41 & $41.8 \%$ \\
Masculino & 98 & $100.0 \%$ \\
Total & \\
\hline
\end{tabular}

En la tabla 1, se puede observar los datos de los adolescentes del nivel secundario de la I.E. 3055 “Túpac Amaru”, siendo la edad promedio 15.76 años distribuidos en 58.2\% corresponde al sexo femenino, $58.2 \%$ y al sexo masculino el $41.8 \%$.

\section{Tabla 2}

Frecuencia de distimia en los adolescentes del nivel secundario

\begin{tabular}{lcc}
\hline \multicolumn{1}{c}{ Distimia } & $\mathrm{N}$ & $\%$ \\
\hline $\mathrm{Si}$ & 58 & $59.2 \%$ \\
No & 33 & $33.7 \%$ \\
Depresión Mayor & 7 & $7.1 \%$ \\
Total & 98 & $100.0 \%$ \\
\hline
\end{tabular}

Fuente: Vivar et al. (2005)

En la tabla 2, se advierte que el 59.2\% de adolescentes de nivel secundario de la I.E. 3055 "Túpac Amaru" presenta distimia y el $33.7 \%$ no la presenta; cabe resaltar que dentro de los alumnos que participaron del estudio también se identificó un $7.1 \%$ que presentó un diagnóstico de depresión mayor. 


\section{Tabla 3}

Situaciones familiares del adolescente y el desarrollo de distimia

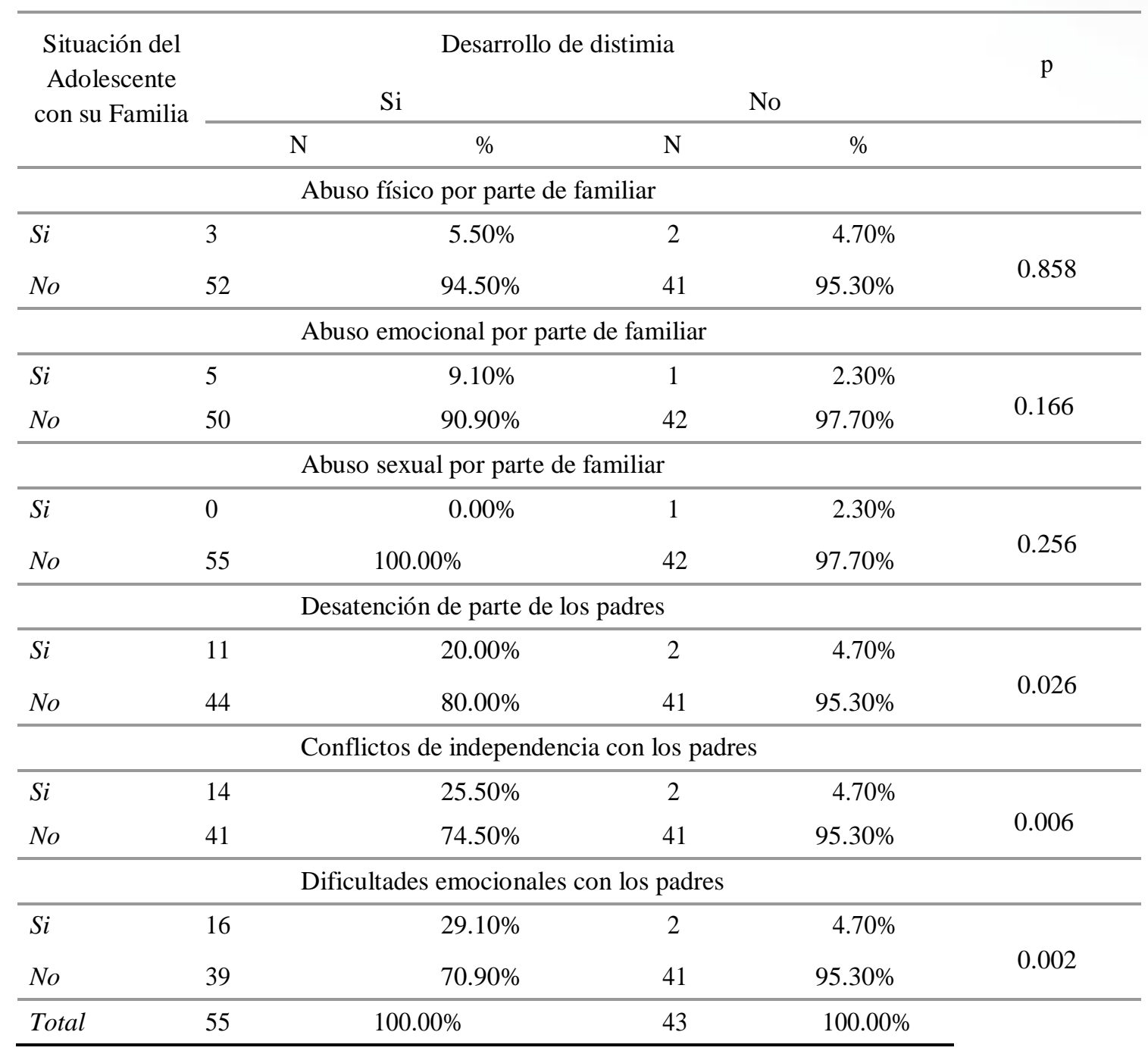

En la tabla 3, se observa la situación de los adolescentes con su familia, según la presencia de distimia, donde:

El 20\% de los adolescentes que refieren desatención de parte de los padres desarrolló distimia y el $4.7 \%$ de la población con el mismo problema no presentó distimia, siendo esta diferencia estadísticamente significativa ( $\mathrm{p}=0.026$ ), es decir la desatención de parte de los padres se relaciona con la presencia de distimia. El 25.5\% que tiene conflictos de independencia con los padres tuvo problemas de distimia y el $4.7 \%$ de adolescentes con la misma dificultad no desarrolló distimia, observándose diferencias significativas ( $\mathrm{p}=0.006$ ), es decir los conflictos de independencia con los padres se relacionan con la presencia de distimia. El $29.1 \%$ de los adolescentes con distimia y el $4.7 \%$ sin distimia presentan dificultad emocional con los padres, 
observándose diferencias estadísticamente significativas ( $\mathrm{p}=0.002)$ en ambos grupos, es decir la dificultad emocional con los padres se relaciona con la presencia de distimia. Respecto al abuso físico $(\mathrm{p}=0.858)$, emocional $(\mathrm{p}=0.166)$ o sexual $(\mathrm{p}=0.256)$ por parte de algún familiar, no se relacionan con la presencia de distimia en los adolescentes.

\section{Tabla 4}

Características de los padres y familia y el desarrollo de distimia de los adolescentes del nivel secundario

\begin{tabular}{|c|c|c|c|c|c|}
\hline \multirow{3}{*}{$\begin{array}{l}\text { Características de } \\
\text { los padres y familia }\end{array}$} & \multicolumn{4}{|c|}{ Desarrolló de distimia } & \multirow{3}{*}{$\mathrm{p}$} \\
\hline & \multicolumn{2}{|c|}{$\mathrm{Si}$} & \multicolumn{2}{|c|}{ No } & \\
\hline & $\mathrm{N}$ & $\%$ & $\mathrm{~N}$ & $\%$ & \\
\hline \multicolumn{6}{|l|}{$\begin{array}{l}\text { Estado civil de los } \\
\text { Padres }\end{array}$} \\
\hline Solteros & 12 & $21.8 \%$ & 8 & $18.6 \%$ & \multirow{4}{*}{0.262} \\
\hline Convivientes & 20 & $36.4 \%$ & 24 & $55.8 \%$ & \\
\hline Casados & 19 & $34.5 \%$ & 9 & $20.9 \%$ & \\
\hline Divorciados & 4 & $7.3 \%$ & 2 & $4.7 \%$ & \\
\hline \multicolumn{6}{|c|}{$\begin{array}{l}\text { Padres con antecedentes } \\
\text { de depresión }\end{array}$} \\
\hline$S i$ & 7 & $12.7 \%$ & 1 & $2.3 \%$ & \multirow{2}{*}{0.062} \\
\hline No & 48 & $87.3 \%$ & 42 & $97.7 \%$ & \\
\hline \multicolumn{6}{|c|}{$\begin{array}{l}\text { Padre con problemas de } \\
\text { Alcoholismo }\end{array}$} \\
\hline$S i$ & 7 & $12.7 \%$ & 1 & $2.3 \%$ & \multirow{2}{*}{0.062} \\
\hline No & 48 & $87.3 \%$ & 42 & $97.7 \%$ & \\
\hline \multicolumn{6}{|c|}{$\begin{array}{l}\text { Madre con problemas de } \\
\text { Alcoholismo }\end{array}$} \\
\hline$S i$ & 2 & $3.6 \%$ & 0 & $0 \%$ & \multirow{2}{*}{0.206} \\
\hline No & 53 & $96.4 \%$ & 43 & $100.0 \%$ & \\
\hline \multicolumn{6}{|c|}{$\begin{array}{l}\text { Ambos padres con problemas } \\
\text { de Alcoholismo }\end{array}$} \\
\hline$S i$ & 2 & $3.6 \%$ & 0 & $0 \%$ & \multirow{2}{*}{0.206} \\
\hline No & 53 & $96.4 \%$ & 43 & $100 \%$ & \\
\hline \multicolumn{6}{|l|}{ Conflictos conyugales } \\
\hline$S i$ & 18 & $32.7 \%$ & 4 & $9.3 \%$ & \multirow{2}{*}{0.006} \\
\hline No & 37 & $67.3 \%$ & 39 & $90.7 \%$ & \\
\hline \multicolumn{6}{|l|}{ Padres fuera de casa } \\
\hline$S i$ & 15 & $27.3 \%$ & 8 & $18.6 \%$ & \multirow{2}{*}{0.315} \\
\hline No & 40 & $72.7 \%$ & 35 & $81.4 \%$ & \\
\hline \multicolumn{6}{|l|}{$\begin{array}{l}\text { Divorcio o separación } \\
\text { conflictiva de padres }\end{array}$} \\
\hline$S i$ & 11 & $20.0 \%$ & 7 & $16.3 \%$ & \multirow{2}{*}{0.637} \\
\hline No & 44 & $80.0 \%$ & 36 & $83.7 \%$ & \\
\hline
\end{tabular}




\section{Tabla 4}

Características de los padres y familia y el desarrollo de distimia de los adolescentes del nivel secundario (continuación)

\begin{tabular}{|c|c|c|c|c|c|}
\hline \multirow{3}{*}{$\begin{array}{l}\text { Características de } \\
\text { los padres y familia }\end{array}$} & \multicolumn{4}{|c|}{ Desarrolló de distimia } & \multirow{3}{*}{$P$} \\
\hline & \multicolumn{2}{|c|}{$\mathrm{Si}$} & \multicolumn{2}{|c|}{ No } & \\
\hline & $\mathrm{N}$ & $\%$ & $\mathrm{~N}$ & $\%$ & \\
\hline \multicolumn{6}{|c|}{ Eventos vitales negativos } \\
\hline$S i$ & 10 & $18.2 \%$ & 0 & $0.0 \%$ & \multirow{2}{*}{0.003} \\
\hline No & 45 & $81.8 \%$ & 43 & $100.0 \%$ & \\
\hline \multicolumn{6}{|c|}{ Muerte de algún familiar } \\
\hline$S i$ & 22 & $40.0 \%$ & 18 & $41.9 \%$ & \multirow{2}{*}{0.852} \\
\hline No & 33 & $60.0 \%$ & 25 & $58.1 \%$ & \\
\hline \multicolumn{6}{|c|}{$\begin{array}{l}\text { Conflictos legales en la } \\
\text { Familia }\end{array}$} \\
\hline$S i$ & 6 & $11.1 \%$ & 2 & $4.7 \%$ & \multirow{2}{*}{0.251} \\
\hline No & 48 & $88.9 \%$ & 41 & $95.3 \%$ & \\
\hline \multicolumn{6}{|c|}{$\begin{array}{l}\text { Intento suicida de algún } \\
\text { Familiar }\end{array}$} \\
\hline$S i$ & 7 & $12.7 \%$ & 0 & $0,0 \%$ & \multirow{2}{*}{0.015} \\
\hline No & 48 & $87.3 \%$ & 43 & $100,0 \%$ & \\
\hline \multicolumn{6}{|l|}{ Violencia familiar } \\
\hline$S i$ & 11 & $20.0 \%$ & 0 & $0.0 \%$ & \multirow{2}{*}{0.002} \\
\hline No & 44 & $80.0 \%$ & 43 & $100.0 \%$ & \\
\hline \multicolumn{6}{|c|}{$\begin{array}{l}\text { Trastorno psiquiátrico de } \\
\text { algún familiar }\end{array}$} \\
\hline$S i$ & 5 & $9.1 \%$ & 2 & $4.8 \%$ & \multirow{2}{*}{0.414} \\
\hline No & 50 & $90.9 \%$ & 40 & $95.2 \%$ & \\
\hline \multicolumn{6}{|c|}{ Cuentan con vivienda propia } \\
\hline$S i$ & 21 & $38.2 \%$ & 25 & $58.1 \%$ & \multirow{2}{*}{0.049} \\
\hline No & 34 & $61.8 \%$ & 18 & $41.9 \%$ & \\
\hline \multicolumn{6}{|c|}{$\begin{array}{l}\text { Desarrollo del hogar en } \\
\text { áreas desfavorecidas }\end{array}$} \\
\hline No & 44 & $80.0 \%$ & 36 & $83.7 \%$ & \multirow{2}{*}{0.637} \\
\hline$S i$ & 11 & $20.0 \%$ & 7 & $16.3 \%$ & \\
\hline \multicolumn{6}{|c|}{$\begin{array}{l}\text { Conflictos familiares por } \\
\text { ingresos económicos bajos }\end{array}$} \\
\hline No & 36 & $65.5 \%$ & 34 & $79.1 \%$ & \multirow{2}{*}{0.149} \\
\hline$S i$ & 19 & $34.5 \%$ & 9 & $20.9 \%$ & \\
\hline Total & 55 & $100.0 \%$ & 43 & $100.0 \%$ & \\
\hline
\end{tabular}

En la tabla 4, se observan las características de los padres y la familia según el desarrollo de distimia, donde:

El 32.7\% de los adolescentes con conflictos conyugales de los padres ha desarrollado distimia 
y el $9.3 \%$ de los adolescentes con el mismo problema no tiene distimia, evidenciándose diferencias significativas ( $\mathrm{p}=0.006$ ), es decir que existe relación entre los conflictos conyugales de los padres y el desarrollo de distimia en los adolescentes.

El $18.2 \%$ de los adolescentes que han experimentado eventos negativos familiares presentó distimia, a diferencia del grupo sin distimia, donde no se registraron casos de adolescentes que hayan pasado por eventos vitales negativos en la familia; observándose que existe relación entre los eventos vitales negativos y la distimia $(\mathrm{p}=0.003)$.

En el $12.7 \%$ de los adolescentes que tuvo algún familiar que intentó suicidarse presentó problemas de distimia, $(\mathrm{p}=0.015)$, de esta tabla se evidencia que existe relación entre el intento suicida de algún familiar y el desarrollo de distimia en los adolescentes.

El 20\% del grupo de adolescentes que han manifestado violencia familiar ha desarrollado distimia. No se encontraron casos de violencia sin desarrollo de distimia; de esta evaluación se deduce que existe diferencia estadísticamente significativa $(\mathrm{p}=0.002)$, es decir la violencia familiar se relaciona a la distimia en los adolescentes.

El $61.8 \%$ de los adolescentes que no cuenta con vivienda propia desarrolló distimia, comparado con el $41.9 \%$ de adolescentes sin vivienda propia que no desarrolló distimia; evidenciándose que existe relación entre no contar con vivienda propia y la distimia $(\mathrm{p}=0.049)$.

Respecto al estado civil de los padres ( $\mathrm{p}=0.262)$, padres con antecedente de depresión ( $\mathrm{p}=0.062)$, padre con antecedente de alcoholismo $(\mathrm{p}=0.062)$, madre con antecedente de alcoholismo $(\mathrm{p}=0.206)$, ambos padres con antecedente de alcoholismo $(\mathrm{p}=0.206)$, padres fuera de casa $(\mathrm{p}=0.315)$, divorcio o separación conflictiva de padres $(\mathrm{p}=0.637)$, muerte de algún familiar $(\mathrm{p}=0.852)$, conflictos legales en la familia $(\mathrm{p}=0.251)$, trastorno psiquiátrico de algún familiar $(\mathrm{p}=0.414)$, desarrollo del hogar en áreas desfavorecidas ( $\mathrm{p}=0.637)$, conflictos familiares por ingresos económicos bajos ( $\mathrm{p}=0.149$ ), no se relacionan con la distimia en los adolescentes.

Tabla 5

Nivel del clima social familiar y el desarrollo de distimia de los adolescentes del nivel secundario

\begin{tabular}{|c|c|c|c|c|c|}
\hline \multirow{3}{*}{ Clima Social Familiar } & \multicolumn{4}{|c|}{ Distimia } & \multirow{3}{*}{$\mathrm{p}$} \\
\hline & \multicolumn{2}{|c|}{$S i$} & \multicolumn{2}{|c|}{ No } & \\
\hline & $N$ & $\%$ & $N$ & $\%$ & \\
\hline Миу Buеna & 0 & $0 \%$ & 2 & $4.7 \%$ & \multirow{4}{*}{0.017} \\
\hline Buena & 6 & $10.9 \%$ & 11 & $25.6 \%$ & \\
\hline Media & 37 & $67.3 \%$ & 29 & $67.4 \%$ & \\
\hline Mala & 12 & $21.8 \%$ & 1 & $2.3 \%$ & \\
\hline Total & 55 & $100.0 \%$ & 41 & $95.3 \%$ & \\
\hline
\end{tabular}

Fuente: Osorio (2019) 
En la tabla 5, se observa que el clima social familiar de los adolescentes con distimia va desde "Media" (67.3\%) a "Mala" (21.8\%), y en el grupo de adolescentes sin distimia, la categoría va desde "Media" (67.4\%) a "Buena" (25.6\%), siendo la diferencia estadísticamente significativa $(\mathrm{p}=0.017)$, es decir existe relación entre el clima social familiar y el desarrollo de distimia en los adolescentes.

\section{Tabla 6}

Dimensiones del clima social familiar y el desarrollo de distimia de los adolescentes del nivel secundario

\begin{tabular}{|c|c|c|c|c|c|}
\hline \multirow{3}{*}{$\begin{array}{l}\text { Dimensiones del Clima } \\
\text { Social Familiar }\end{array}$} & \multicolumn{4}{|c|}{ Distimia } & \multirow{3}{*}{$\mathrm{p}$} \\
\hline & \multicolumn{2}{|c|}{$S i$} & \multicolumn{2}{|c|}{$\mathrm{No}$} & \\
\hline & $N$ & $\%$ & $N$ & $\%$ & \\
\hline \multicolumn{6}{|c|}{ Relaciones } \\
\hline Миу Buеna & 5 & $9.1 \%$ & 14 & $32.6 \%$ & \multirow{5}{*}{0.008} \\
\hline Buena & 9 & $16.3 \%$ & 4 & $9.3 \%$ & \\
\hline Media & 19 & $34.5 \%$ & 19 & $44.2 \%$ & \\
\hline Mala & 12 & $21.8 \%$ & 3 & $7.0 \%$ & \\
\hline Muy mala & 10 & $18.2 \%$ & 3 & $7.0 \%$ & \\
\hline \multicolumn{6}{|c|}{ Desarrollo } \\
\hline Миу Buеna & 1 & $1.8 \%$ & 3 & $7.0 \%$ & \multirow{5}{*}{0.030} \\
\hline Buena & 6 & $10.9 \%$ & 13 & $30.2 \%$ & \\
\hline Media & 12 & $21.8 \%$ & 13 & $30.2 \%$ & \\
\hline Mala & 17 & $30.9 \%$ & 8 & $18.6 \%$ & \\
\hline Muy mala & 19 & $34.6 \%$ & 6 & $14.0 \%$ & \\
\hline \multicolumn{6}{|c|}{ Estabilidad } \\
\hline Buena & 0 & $0.0 \%$ & 2 & $4.7 \%$ & \multirow{4}{*}{0.047} \\
\hline Media & 29 & $52.7 \%$ & 28 & $65.1 \%$ & \\
\hline Mala & 17 & $30.9 \%$ & 12 & $27.9 \%$ & \\
\hline Muy mala & 9 & $16.4 \%$ & 1 & $2.3 \%$ & \\
\hline Total & 55 & $100.0 \%$ & 43 & $100.0 \%$ & \\
\hline
\end{tabular}

Fuente: Osorio (2019)

En la tabla 6, se evalúan las dimensiones del clima social familiar según la distimia en los adolescentes, donde:

Respecto a la dimensión relaciones del clima social familiar, el 34.5\% y $21.8 \%$ de los adolescentes con distimia presentaron categorías de "Media" y "Mala", respectivamente, mientras que los adolescentes sin distimia presentaron categorías "Media" (44.2\%) y "Muy Buena" (32.6\%); del estudio se evidencia que existe diferencia significativa entre ambas variables $(\mathrm{p}=0.008)$. 
Acerca de la dimensión "desarrollo" del clima social familiar, los adolescentes que presentaron distimia tuvieron niveles de "Muy Mala" (34.6\%) a "Mala" (30.9\%), mientras que, en el grupo de adolescentes sin distimia, la categoría es desde "Buena" (30.2\%) a "Media" (30.2\%), observándose diferencias estadísticamente significativas $(\mathrm{p}=0.030)$.

En la dimensión "Estabilidad" del clima social familiar, el 52.7\% de los adolescentes con distimia tuvo una categoría "Media" y los adolescentes sin desarrollo de distimia, tuvieron una categoría "Media" (65.1\%), siendo esta diferencia significativa ( $\mathrm{p}=0.047)$.

\section{Tabla 7}

Grado de adaptabilidad a los cambios externos e internos en la familia y el desarrollo de distimia de los adolescentes del nivel secundario

\begin{tabular}{|c|c|c|c|c|c|}
\hline \multirow{3}{*}{$\begin{array}{l}\text { Grado de } \\
\text { Adaptabilidad a cambios } \\
\text { externos e internos de } \\
\text { una familia }\end{array}$} & \multicolumn{4}{|c|}{ Distimia } & \multirow{3}{*}{$p$} \\
\hline & \multicolumn{2}{|c|}{$\mathrm{Si}$} & \multicolumn{2}{|c|}{ No } & \\
\hline & $\mathrm{N}$ & $\%$ & $\mathrm{~N}$ & $\%$ & \\
\hline Rígida & 2 & $3.6 \%$ & 1 & $2.3 \%$ & \multirow{4}{*}{0.283} \\
\hline Estructurada & 11 & $20.0 \%$ & 3 & $7.0 \%$ & \\
\hline Flexible & 10 & $18.2 \%$ & 11 & $25.6 \%$ & \\
\hline Caótica & 32 & $58.2 \%$ & 28 & $65.1 \%$ & \\
\hline Total & 55 & $100.0 \%$ & 43 & $100.0 \%$ & \\
\hline
\end{tabular}

Fuente: Del Valle (2006)

En la tabla 7, se observa que el 58.2\% de los adolescentes con desarrollo de distimia presenta un grado de adaptabilidad "Caótica" y en los adolescentes sin distimia el grado de adaptabilidad es "Caótica" en el 65.1\%, no observándose diferencias significativas en ambos grupos ( $\mathrm{p}=0.283)$.

\section{Tabla 8}

Grado de cohesión a los cambios externos e internos en la familia y el desarrollo de distimia de los adolescentes del nivel secundario

\begin{tabular}{|c|c|c|c|c|c|}
\hline \multirow{3}{*}{$\begin{array}{l}\text { Grado de cohesión a cambios } \\
\text { externos e internos de una } \\
\text { familia }\end{array}$} & \multicolumn{4}{|c|}{ Distimia } & \multirow{3}{*}{$p$} \\
\hline & \multicolumn{2}{|c|}{$\mathrm{Si}$} & \multicolumn{2}{|c|}{ No } & \\
\hline & $\mathrm{N}$ & $\%$ & $\mathrm{~N}$ & $\%$ & \\
\hline No relacionada & 33 & $60.0 \%$ & 25 & $58.1 \%$ & \multirow{4}{*}{0.885} \\
\hline Semi-relacionada & 17 & $30.9 \%$ & 12 & $27.9 \%$ & \\
\hline Relacionada & 3 & $5.5 \%$ & 4 & $9.3 \%$ & \\
\hline Aglutinada & 2 & $3.6 \%$ & 2 & $4.7 \%$ & \\
\hline Total & 55 & $100.0 \%$ & 43 & $100.0 \%$ & \\
\hline
\end{tabular}

Fuente: Del Valle (2006) 
En la tabla 8, se observa que el $60 \%$ de los adolescentes con desarrollo de distimia presenta un grado de cohesión "No relacionada" y en los adolescentes sin el desarrollo de este trastorno, el grado de cohesión es "No relacionada" en el $58.1 \%$ de los casos; del estudio se deduce que no existe diferencia significativa en ambos grupos $(\mathrm{p}=0.885)$.

\section{Conclusiones}

- En relación con los factores relacionales familiares y el desarrollo de la distimia, se identificó que la desatención de parte de los padres se encuentra asociada con el desarrollo de este trastorno; puesto que la familia no presta la atención requerida por el adolescente, justamente en esta etapa, en la que el ser humano necesita más de este soporte. Es importante mencionar que la familia tiene un impacto perdurable sobre los problemas conductuales del niño y del adolescente. Por otro lado, se ha visto que los conflictos de independencia con los padres y la dificultad emocional con los mismos también se asocian de forma muy significativa con la presencia de distimia. Es menester señalar que el sistema relacional de una familia en la etapa adolescente se enfrenta a intensos cambios en uno o más de sus miembros $\mathrm{y}$, por lo tanto, necesariamente también cambian su propio funcionamiento, desencadenando en patrones comportamentales parenterales que desequilibran su estructura. En su mayoría los adolescentes presentan distimia por una independencia de los padres e igualmente por dificultades emocionales, lo que conlleva a que tengan un comportamiento melancólico, a este respecto Korman y Sarudiansky (2011) ha encontrado que se trata de padecimiento que se manifiesta en personas muy pesimistas y negativas, pero que continúan con sus actividades sociales y laborales.

- En el presente estudio, se identificó una relación significativa, entre los conflictos conyugales de los padres y el desarrollo de distimia en los adolescentes. Esta evidencia es sustentada por Linares (2013), quien destaca las características de la familia de un distímico, las cuales se basan en que los cónyuges se ven enfrentados, generando un conflicto, lo cual arrastra al hijo a formar alianza con uno de ellos y a tomar una posición contraria por el otro. La coalición con uno de los progenitores se asocia pronto a la pérdida de la relación con el otro, provocando tristeza, la cual se expresará más adelante, cuando la vida genere nuevas pérdidas relacionalmente significativas, traduciéndose en el conglomerado de síntomas ansioso-depresivos, típicamente neuróticos, que caracterizan a la distimia.

- Se concluye también que, el $12.7 \%$ de los adolescentes que tuvo algún familiar que intentó suicidarse presento problemas de distimia, demostrando así que existe relación entre el intento suicida de algún familiar y el desarrollo de distimia en los adolescentes. El tener antecedente familiar con intento de suicidio no ha sido relacionado a la distimia de manera directa por otros autores, 
solo Nobile et al. (2003), menciona que las interacciones familiares anormales incrementan el riesgo de aparición de un trastorno distímico y el intento de suicidio de un familiar que forma parte de su entorno más cercano podría influir el desarrollo distímico.

- Acerca de la violencia familiar, se determinó que el $20 \%$ de los adolescentes que presentó violencia familiar desarrolló distimia, en cambio, de los adolescentes que tuvieron distimia, ninguno ha vivido violencia familiar, lo cual tiene cierta semejanza con lo reportado por otros trabajos, un ejemplo de ello es el realizado por Serfaty et al. (2001), quienes observaron que la violencia familiar es frecuente en los adolescentes de sexo femenino y se asocia con el diagnóstico de distimia. Los resultados manifiestan que el adolescente que convive en un hogar donde hay violencia puede generar ansiedad, depresión y distimia, haciéndose notar en ciertos síntomas como desgano, insomnio, dolores de cabeza, falta de concentración, etc.

\section{Referencias Bibliográficas}

American Psychiatric Association [APA]. (2014). Manual Diagnostico y Estadístico de los Trastornos Mentales DSM-5. Madrid-España: Médica Panamericana.

Cassullo, G. (1998). Algunas Consideraciones Acerca del Concepto de Clima Social y su Evaluación. Buenos Aires: Universidad de Buenos Aires.

Del Valle, G. (2006). Uso de la Escala de Evaluación de Adaptabilidad y Cohesión Familiar (FACES III) en población clínica de un equipo de intervención en crisis. XIII Jornadas de Investigación y Segundo Encuentro de Investigadores en Psicología del Mercosur. Facultad de Psicología - Universidad de Buenos Aires, Buenos Aires, 2006., (págs. 36-38). Buenos Aires - Argentina.

Galicia, I., Sánchez, A., \& Robles, F. (diciembre de 2009). Factores asociados a la depresión en adolescentes: Rendimiento escolar y dinámica familiar. Anales de Psicología, 25(2), 227-240.

Hoyos, E., Lemos, M., \& Torres, Y. (enero - junio de 2012). Factores de Riesgo y de Protección de la Depresión en los Adolescentes de la Ciudad de Medellín.

Korman, G., \& Sarudiansky, M. (2011). Modelos Teóricos y Clínicos para la Conceptualización y Tratamiento de la Depresión. Subjetividad y Procesos Cognitivos UCES, 15(1), 119-145.

Linares, J. (2013). Una psicopatología relacional es posible. Revista Mexicana De Investigación En Psicología, 5(2), 189-196. 
Ministerio de Salud Gobierno de Chile. (2013). Guía clínica para el tratamiento de adolescentes de 10 a 14 años con Depresión. Santiago-Chile.

Ministerio de Salud Pública Gobierno del Perú. (2014). Boletín Epidemiológico. LimaPerú.

Nobile, M., Cataldo, G., Marino, C., \& Molteni, M. (2003). Diagnosis and Treatment of Dysthymia in Children and Adolescents. CNS Drugs, 927-946.

Organización Panamericana de la Salud [OPS]. (2012). Día Mundial de la Salud Mental: la depresión es el trastorno mental más frecuente. Washington, DC-United States of America.

Osorio, V. (2019). Clima Social Familiar y su Relación con la Depresión en estudiantes de secundaria de una Institución Educativa. Chiclayo, 2018. CHICLAYO PERÚ: Universidad Particular de Chiclayo.

Pérez, J. (2020). Distimia. Bogotá D.C.-Colombia: Universidad de los Andes.

Rafael, A. (2007). Escalas de evaluación II: escalas para los trastornos internalizados. Centre Londres 94, 5.

Serfaty, E., Casanueva, E., Zavala, M., Andrade, J., Boffi, H., Leal, M., \& Foglia, V. (diciembre de 2001). Violencia y riesgos asociados en adolescentes. Revista Argentina de Clínica Neuropsiquiátrica, 10(3).

Vivar, R., Pacheco, Z., Adrianzen, C., Macciotta, B., \& Marchena, C. (2005). Validación de la Escala de Birleson Modificada para Trastornos Depresivos en Niños y Adolescentes Peruanos. Revista Peruana De Pediatría, 24-30.

Wan, D. (2012). Depression: A Global Crisis. World Federation for Mental Health, 2-29.

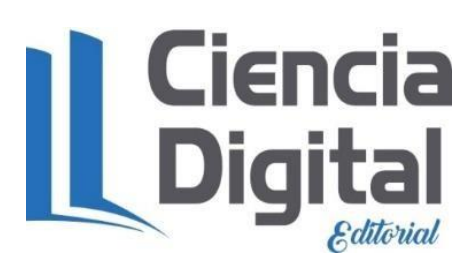


El artículo que se publica es de exclusiva responsabilidad de los autores y no necesariamente reflejan el pensamiento de la Revista Conciencia Digital.

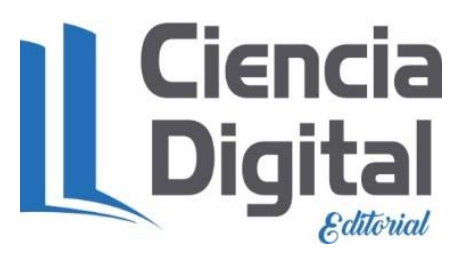

El artículo queda en propiedad de la revista y, por tanto, su publicación parcial y/o total en otro medio tiene que ser autorizado por el director de la Revista Conciencia Digital.
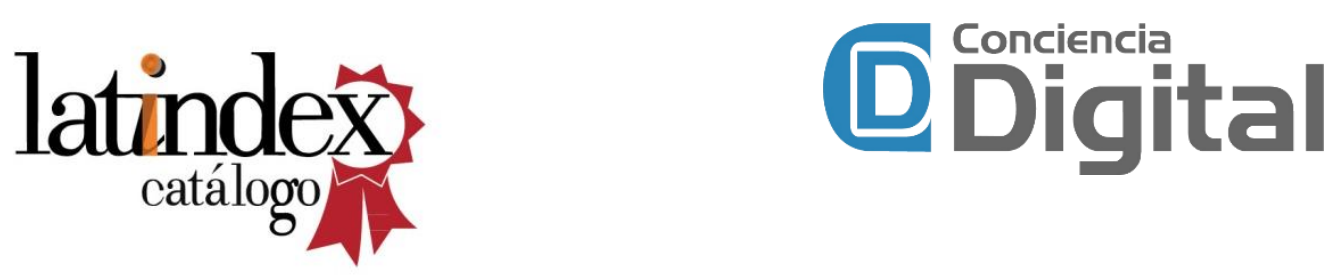

Indexaciones

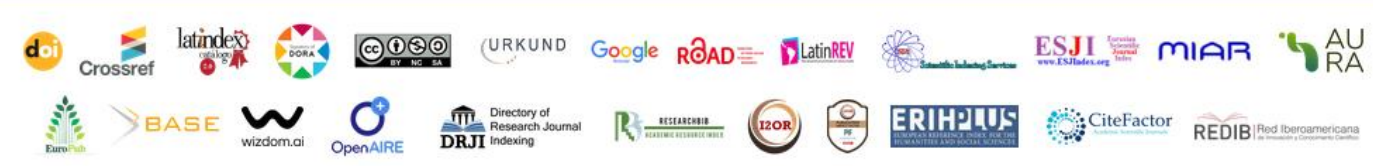

